\title{
School-based interventions targeting stigma of mental illness: systematic review
}

\author{
Catriona Mellor ${ }^{1}$ \\ ${ }^{1}$ Oxford Health NHS Foundation Trust, \\ Oxford, UK \\ Correspondence to Catriona Mellor \\ (catriona.mellor@oxfordhealth.nhs.uk) \\ First received 9 Oct 2012, final revision \\ 24 Nov 2013, accepted 2 Dec 2013 \\ (C) 2014 The Royal College of \\ Psychiatrists. This is an open-access \\ article published by the Royal College \\ of Psychiatrists and distributed \\ under the terms of the Creative \\ Commons Attribution License (http:// \\ creativecommons.org/licenses/by/ \\ 3.0), which permits unrestricted use, \\ distribution, and reproduction in any \\ medium, provided the original work is \\ properly cited.
}

Psychiatric Bulletin (2014), 38, 164-171, doi: 10.1192/pb.bp.112.041723

\begin{abstract}
Aims and method To systematically review the published literature on the effectiveness of classroom-based interventions to tackle the stigma of mental illness in young people, and to identify any consistent elements within successful programmes.
\end{abstract}

Results Seventeen studies were included in the analysis. A minority of studies reported a positive impact on stigma or knowledge outcomes at follow-up and there were considerable methodological shortcomings in the studies reviewed. These interventions varied substanitally in content and delivery. It was not possible to use this data to draw out what aspects make a successful intervention. There is currently no strong evidence to support previous conclusions that these types of intervention work for children and adolescents.

Clinical implications When anti-stigma interventions for young people are rolled out in the future, it is important that the programme design and method of delivery have evidence to prove their effectiveness, and that the audience and setting are the most appropriate to target. There is a current lack of strong evidence to inform this.

\section{Declaration of interest None.}

It is estimated that in high-income countries $5-20 \%$ of children and adolescents require mental health services and, in Europe, provision of services to those in need can be as low as $20 \%$. $^{1}$ There is growing evidence that barriers to seeking help and achieving recovery for mental health problems include the stigma around mental illness, ${ }^{2,3}$ and that stigmatising attitudes start young. ${ }^{4}$ Stigmatising attitudes to mental illness are widespread. ${ }^{5}$ There is national and international recognition that this issue should be prioritised. ${ }^{6,7}$ There is mixed evidence as to whether national initiatives can change such attitudes. ${ }^{8-11}$ Attempts to research and implement school-based mental health promotion in the UK have largely focused on making the whole-school environment more emotionally aware. ${ }^{12-15}$ However, there are also many initiatives, in the UK and other countries, taking anti-stigma programmes into the classroom (for example Rethink, Royal College of Psychiatrists). One review concluded that educational interventions in schools provide positive outcomes on pupils' attitudes to mental illness, ${ }^{16}$ a belief that seems widespread in the literature. However, it reports positive findings of the studies reviewed without clearly describing the quality of the studies, making the findings difficult to interpret. Its conclusions are considerably more optimistic than an earlier review that questioned the reliability and validity of all studies reviewed. ${ }^{17}$ However, there have been many published studies since 2006 (the limit of Schachter's et al's meticulous report ${ }^{17}$ ), which may explain the discrepancy. This systematic review addresses two specific questions: (a) what current evidence is there to justify the growing optimism as to the effectiveness of school-based anti-stigma programmes and (b) what evidence is there to inform future successful programme design?

\section{Method}

\section{Inclusion criteria}

The types of studies included (using Cochrane Effective Practice and Organisation of Care (EPOC) group definitions) were randomised controlled trials (RCTs), cluster RCTs, non-randomised controlled trials (NRTs), or controlled before-after studies (CBA). Participants were children or adolescents attending primary or secondary school. Schoolbased interventions targeting attitudes and stigma about mental illness were included. Studies were included if they measured outcomes of: knowledge/beliefs and attitudes towards mental illness, behavioural intentions, stigmatising behaviour or affect. The analysis of help-seeking outcomes is not covered in this review, because help-seeking is not directly associated with stigmatising attitudes/behaviour. Level of knowledge is also not directly associated with stigmatising attitudes but these outcomes are included as many of the 'knowledge' measures contain some belief and attitude statements. Known reliability/validity of the instruments was not an inclusion criterion, but will be commented on within the results. 


\section{Search methods and study selection}

The following search engines were used: Medline, CINAHL and PsycINFO (1990-2013, articles in English) on 12 June 2013, using the keywords (stigma* OR attitude* OR awareness) AND (school or adolesc*) AND (educat* OR train* OR program*) AND (mental OR schizophreni* OR psychiatri*). The references lists cited in relevant reviews were also checked. ${ }^{16-21}$ Studies were selected for inclusion by screening titles, abstracts and when necessary full texts, against the inclusion criteria.

\section{Data extraction and critical appraisal}

A data-extraction form based on the Cochrane EPOC group's data-collection checklist was used to record details about study characteristics, intervention design, outcome measures and results. Following this process the group's recently updated 'suggested risk of bias criteria for EPOC reviews ${ }^{22}$ was used to make judgements on the risk of bias (high, low or unclear) in each study in each of the domains suggested by the document. The domains assessed were: allocation sequence generation and concealment, baseline outcome measures and characteristics, comparison of site profiles (if applicable), protection against contamination, masking, completeness of outcome data, and outcome reporting (were data for each outcome, group and time point fully presented). In addition to this, the reliability and validity of the instruments used, as documented in the study reports, was noted.

\section{Data synthesis}

The review looked at the intervention effect of each study by comparing before and after outcome scores in the intervention and control groups. First, studies that provided follow-up data (rather than simply immediate post-test data) were reviewed. Of these, studies that reported a positive result (a statistically significant, $P<0.05$, change in any outcome measure compared with control) after the intervention were selected. These studies were reviewed for study quality, as judged by study design and risk of bias criteria. Studies with positive results at immediate postintervention only were then reviewed for study quality. Positive results based on the use of specifically developed outcome measures with low reliability were excluded. To answer the second review question the intervention design features (such as duration, contact or non-contact, delivery) of those studies showing positive results were tabulated and compared.

\section{Results}

Of the 1261 studies identified in the initial search, 17 met the above criteria (Fig. 1). ${ }^{23}$

\section{Intervention and study characteristics}

The interventions varied in content and delivery methods (online Table DS1). Nine were education-only, ${ }^{24-32}$ whereas eight had indirect ${ }^{33,34}$ or direct ${ }^{35-40}$ contact with someone with lived experience. Fifteen studies targeted secondaryschool pupils, two targeted primary school pupils. ${ }^{28,31}$ One included a few individuals over $18 .^{35}$ The duration ranged from one-off interventions lasting $30-120 \mathrm{~min}$ to multiple sessions over a period of up to 4 months. The focus of the interventions was mental illness in 11 studies, schizophrenia in 3 and depression in 3 . Five studies investigated the impact of already established interventions. ${ }^{30,36-38,40}$
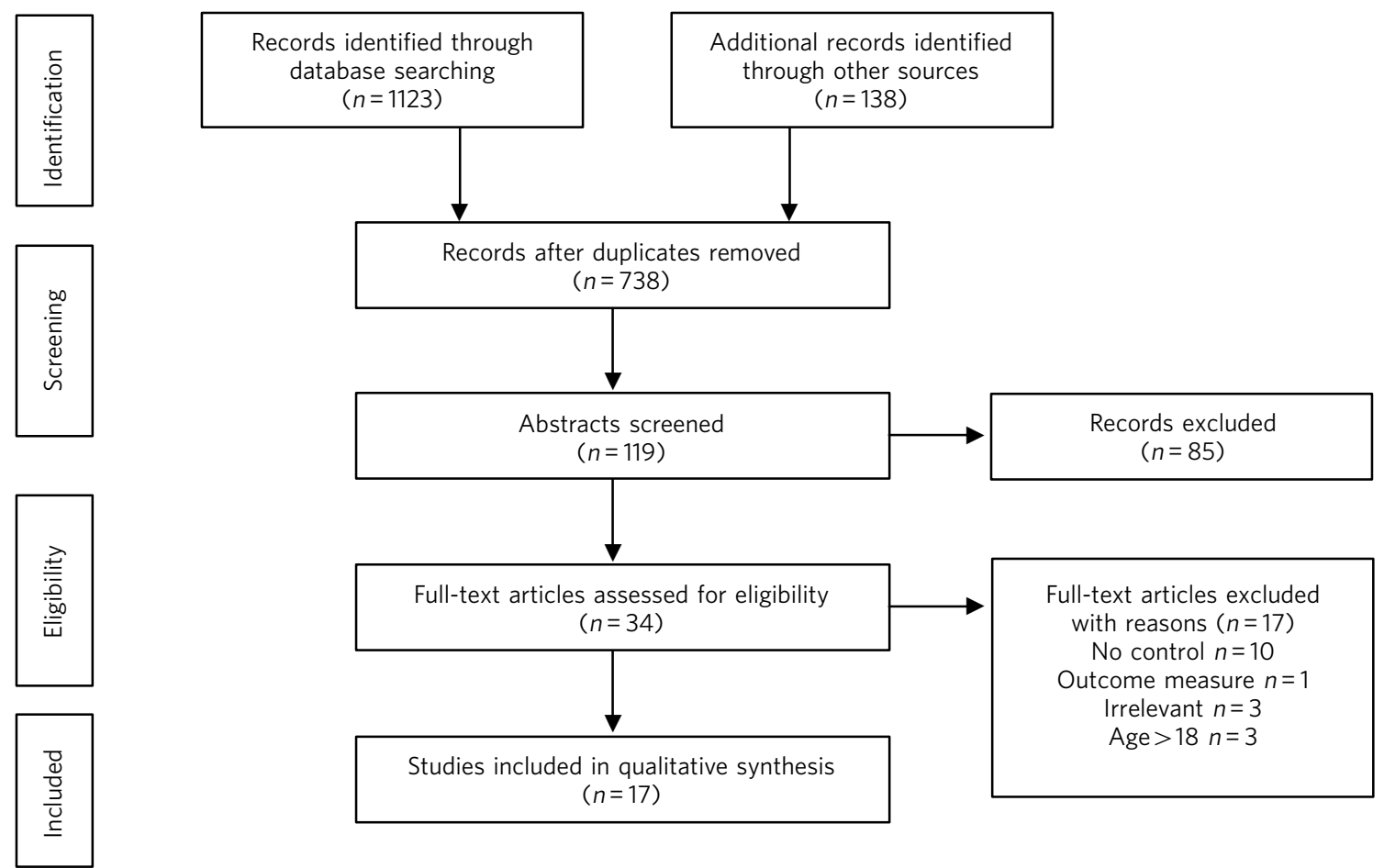

Fig 1 Selection of studies using Preferred Reporting Items for Systematic reviews and Meta-Analyses (PRISMA). 
The number of participants varied from 40 to 616 . The follow-up time ranged from immediately post intervention only, up to 12 months. The outcome measures were secondary outcomes in two studies, ${ }^{26,27}$ which are shown at the end of Table DS1. One study was an RCT. ${ }^{34}$ Five studies were cluster RCTs, two using cluster randomisation at the school level, ${ }^{24,28}$ three using cluster randomisation at the class/year level within selected schools. ${ }^{32,33,40}$ Four studies were NRTs, six were CBA trials. It is unclear whether one study was an NRT or CBA. ${ }^{38}$

The comparison groups, other than Chan et al's, ${ }^{33}$ which compared three intervention conditions, had normal lessons (no intervention) in 14 of the studies, a talk about healthy living from external speakers in $1^{32}$ and a video presentation about smoking in another. ${ }^{34}$ The vast majority therefore did not control for the effect of a novel programme, in many cases with outside speakers.

\section{Outcome measures}

Table DS1 shows all outcome measures used within the studies. Results from two additional scales were excluded as irrelevant to the review question (the Self-Efficacy Scale ${ }^{38}$ and the Strengths and Difficulties Questionnaire $\left.{ }^{29}\right)$. Of the remaining 31 outcome measures used (and reviewed here), most measures were 'stigma' measures: attitudes, behavioural intentions and in one study an affect measure. ${ }^{40}$ In addition, several studies tested factual knowledge gained. No studies measured actual behaviour. All measurements were selfreport Yes/No, True/False or Likert-style questionnaires, except for the Implicit Association Test (IAT), ${ }^{34}$ where participants categorise words as quickly as possible.

For 14 of the instruments reliability was reported as good, in all but one of these cases the studies chose to report internal consistency (Cronbach's alpha) to back up that claim. The instruments' validity was usually not mentioned in the report, although some studies used previously welltested instruments.

A total of 13 of the instruments were designed for the intervention or study; 6 of these had poor (or untested) reliability, casting doubt also on their validity ${ }^{24,29-31,37,40}$ and therefore on the results that they provide. These six were all knowledge measures. The other seven were piloted and/or internally consistent. . $^{30,31,33,36}$

\section{Study quality}

Details of study quality are provided in Table 1. Only one study, a cluster RCT, adequately described randomisation and allocation concealment. ${ }^{24}$ Baseline outcome measures and baseline group characteristics were clearly compared and similar in nine (in addition, one study showed similarity in one but not the other outcome ${ }^{37}$ ) and six studies respectively. Four studies used different sites as their control and intervention groups and none of these studies clearly compared (with measures of significance) the sites' profiles. These four studies only were able to clearly protect against contamination. Due to using self-report questionnaires none of the outcome measures were masked or objective (the IAT is 'self-report' but aims to assess automatic memory associations and therefore is less open to $\operatorname{bias}^{34}$ ). There was the potential of attrition bias being introduced because of incomplete data in 13 studies. Most of the studies did not omit important outcome data in their reports. Four studies mention a power calculation. One was underpowered, ${ }^{40}$ the other three report having sufficient sample size. ${ }^{26,27,29}$

Various methods were used to enhance consistency of delivery. In two studies the presenters were trained and sessions monitored for fidelity ${ }^{29,40}$ and two interventions used a computer program..$^{26,27}$ Five others mention training the presenters, ${ }^{24,33,34,37,38}$ the remaining eight provided material for the presenters to follow.

\section{Intervention effects}

To answer the first review question it is helpful to look at whether the studies with positive changes in stigma (and knowledge) outcomes are of high enough quality to give confidence in their findings. The final two rows of Table 1 show which studies reported statistically significant results at follow-up (for knowledge and stigma outcomes). Results of each outcome measure are tabulated as either reporting a significant positive change (a tick) or no significant change (a cross).

Table 2 gives an overview of the results reported in the studies at post-test and follow-up, and indicates whether the outcomes measured changed significantly (a tick) or not (a cross). Results from the six outcome measures developed specifically for the interventions they were testing, with reliability not measured (or $\alpha<0.7$ ), are not included in this section. Table 2 shows which outcome measures this applies to (represented by /). For two studies, where this involved the only instrument used, ${ }^{24,29}$ there are therefore limited conclusions that can be drawn here despite the fact that they did otherwise have relatively good methodology, according to the risk of bias table.

\section{Studies with positive results at follow-up}

Twelve studies collected information at follow-up. Of these, seven studies showed some statistically significant positive changes at follow-up, ${ }^{25,30-33,35,36}$ and these are summarised here. All were at high risk of selection bias except for the two cluster RCTs, which did not, however, have a clearly described method of randomisation. All had high-risk levels of attrition or an unclear description of individuals who dropped out, except for Economou et $a l^{32}$

Economou et al's ${ }^{32}$ cluster RCT compared change in mean score per item on their belief/attitude questionnaire and reported that $8 / 10$ items were answered significantly better at follow-up than baseline in the intervention group. They report no significant change in the control group but do not present these data. There was no significant improvement in social distance scores at follow-up. ${ }^{32}$ Chan et al's ${ }^{33}$ cluster RCT showed significant positive change in knowledge and social distance but not stigma at follow-up. This study discarded $35 \%$ of their data (because of absenteeism or returning incomplete measures) and it was not clear from which group(s) these missing data were from.

Ventieri et al's ${ }^{31}$ study in a primary school used Schulze et al's ${ }^{36}$ social distance scale and a novel instrument to test 'benevolence' and 'unkindliness', piloted on a group of pre-adolescents and tested for reliability. The intervention group showed positive change compared with the controls 


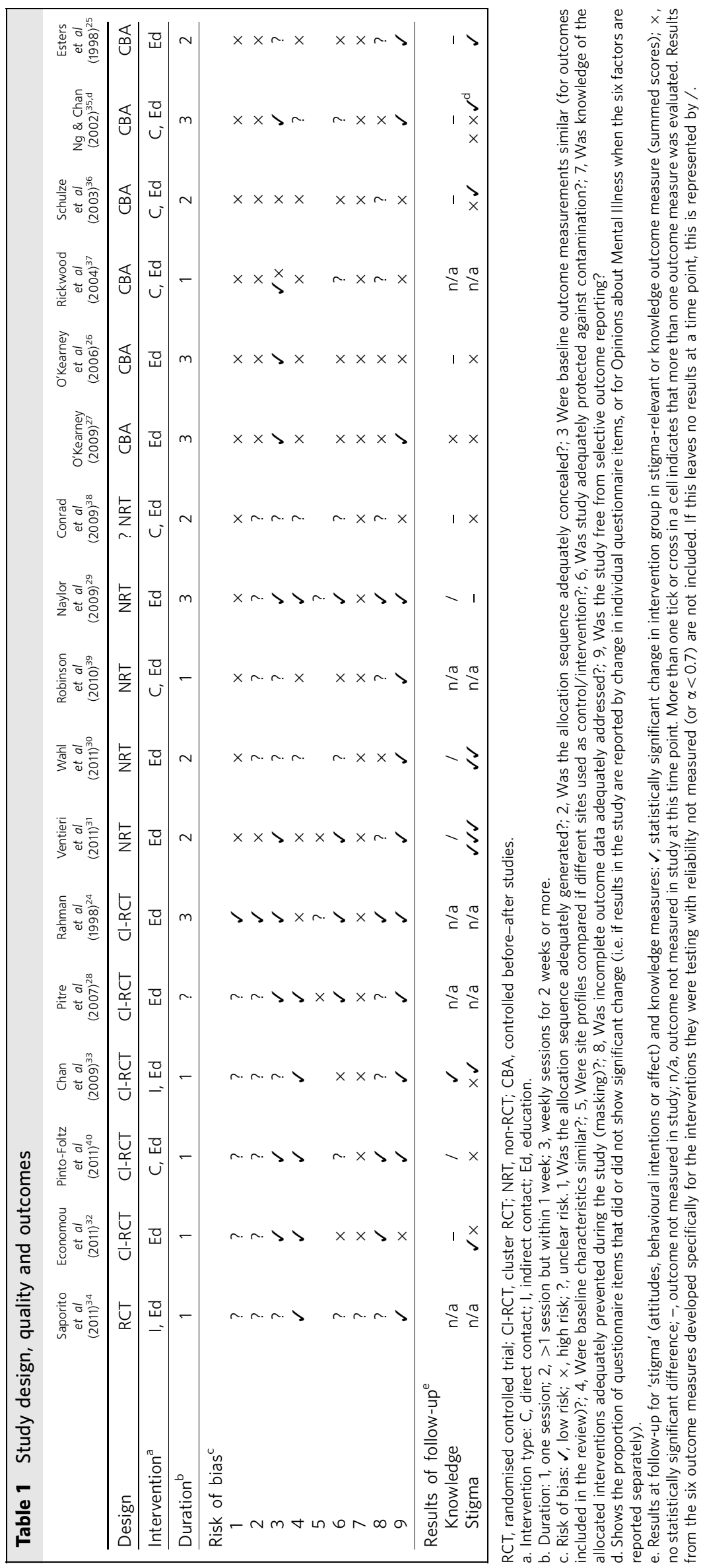




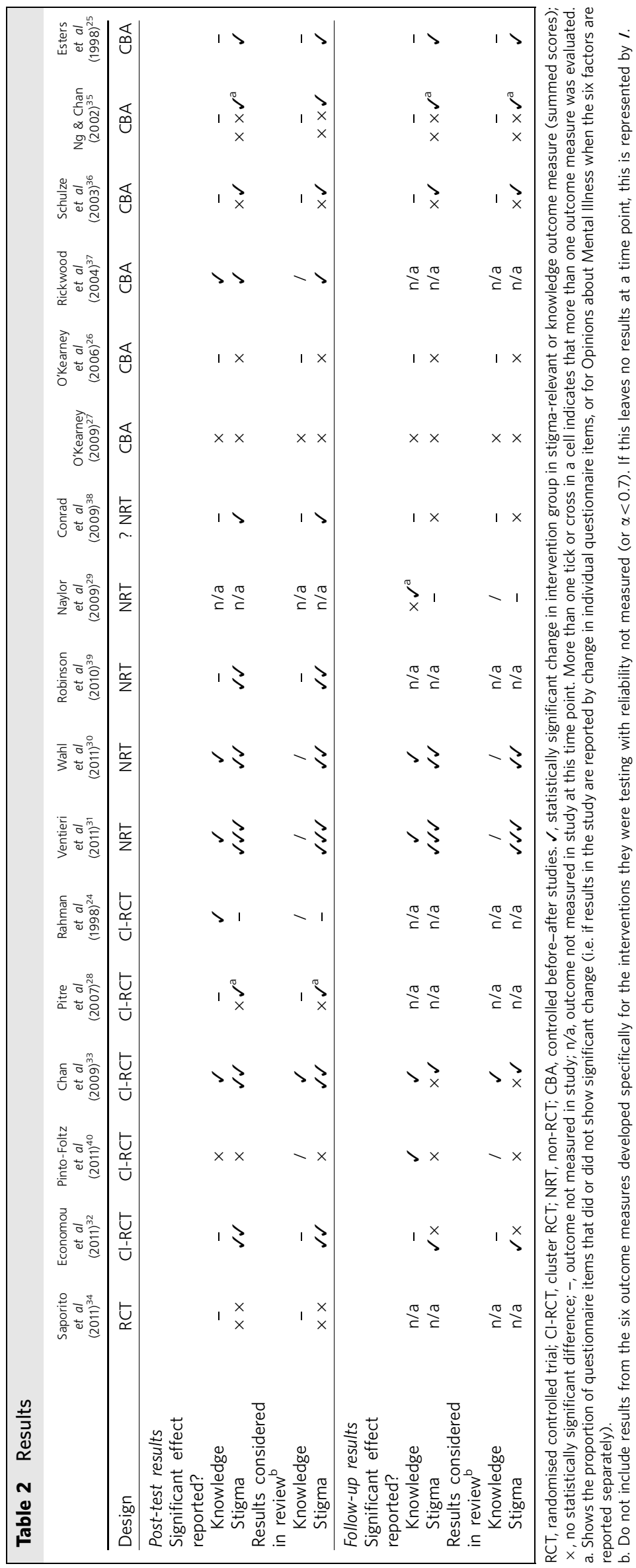


in all three measures. Schools were invited into the study based on assignment (to control or intervention). ${ }^{31}$ In Wahl et $a$ ' $^{30}{ }^{30}$ study, mean total score in knowledge, attitudes and social distance (on scales developed for the study) improved slightly but significantly. Only $47 \%$ of eligible pupils were included in the analysis (those who took part in the three-session programme and completed all questionnaires).

Schulze and colleagues summed the amount of positive responses from each student on their novel instrument testing for stereotypes and social distance. ${ }^{36}$ Stereotypes, but not social distance, changed more positively in the intervention than in the control group. This study reported significant differences in baseline outcome measures and baseline characteristics, likely related to the fact their intervention group chose to take part in the mental illness module. $\mathrm{Ng} \& \mathrm{Chan}^{35}$ report a significant improvement in 2/6 Opinion about Mental Illness in Chinese Community (OMICC) factors (benevolence and stigmatisation) between the intervention and control groups, but a significant worsening in both groups in attitudes to restrictiveness. Esters et al's small study $(n=40)$ reported statistically significant positive change on a well-validated scale measuring opinions about mental illness. ${ }^{25}$

Studies with positive results at post-test but not follow-up There were a further four studies that report statistically significant positive results at immediate post-test only (Table 2). They all have high or unclear risk of selection and attrition bias. Pitre et al's three-session puppet show in a primary school reports positive change for the intervention group on the adapted Opinions about Mental Illness (OMI) scale, in $3 / 6$ factors. $^{28}$ Robinson et al's study reports significant changes (compared with baseline and control) after their $2 \mathrm{~h}$ session on stigma and attitudes. ${ }^{39}$ The studies of Rickwood et $a l^{37}$ (one session intervention) and Conrad et $a l^{38}$ (1-day intervention), do not present any data other than regression statistics, making their findings hard to assess.

\section{No positive results}

Some studies showed no significant changes at either posttest or follow-up. Saporito et $a l^{34}$ was the only RCT, randomising at pupil level, although it is not clear what method of randomisation they used. There was no significant improvement in implicit or explicit attitudes to mentally ill people. Pinto-Foltz et $a l^{40}$ carried out a cluster RCT with more low-risk scores than most of the other studies reviewed. They provided a one-session intervention and found no post-intervention difference in stigmatising attitudes. O'Kearney et al's ${ }^{26}$ and O'Kearney's ${ }^{27}$ studies of five online sessions (one in males, one in females) recorded results at 5 months. Attitudes (and depression literacy in the later study) were measured as secondary outcomes but showed no significant change. ${ }^{7}$

\section{Effective intervention design}

To answer the second review question it is necessary to see whether there are any consistent features in the intervention programmes in those studies that show positive results. However, the comparison between the results of studies describing such different interventions and methodology is difficult. Chan et $a l^{33}$ is the only example of a study investigating which aspect of a one-off session might offer the most benefit. The most improvement was seen in the group that had education (a 30 min lecture) followed by a 15 min video (rather than vice versa, or purely education).

Of the studies with positive results at follow-up there is no obvious pattern about what makes a successful intervention. These seven studies include two interventions of only one session and one of the longest interventions (over 10 weeks). Four had no element of contact, two direct, one indirect contact. The follow-up time at which the positive results were recorded ranged from 1 to 12 months. One study was in a primary school.

\section{Discussion}

Within the literature there are frequent references to the existing evidence for the effectiveness of school-based interventions in reducing stigma of mental illness in young people. This systematic review of available evidence does not support those statements. Showing a significant difference in self-report questionnaires immediately after an intervention seems unsurprising and, if that is the limit of the effect of the programme, seems insufficient grounds for rolling out the programme more widely. It is proposed here that a successful programme would show a positive change in outcomes at follow-up, which was the case in seven studies ${ }^{25,30-33,35,36}$ However, the potential for selection and attrition bias, which can exaggerate intervention effect, are common themes in all but one (Economou et $a l^{32}$ ) of these studies.

There is one RCT and five cluster RCTs within this body of evidence. Only two of these showed statistically significant improvements in outcome measures at followup. Only one of the RCTs clearly described their randomisation process, making it difficult to judge the risk of selection bias in the others. Of the other study designs, Naylor et al's ${ }^{29}$ study stands out as having a greater number of lowrisk scores. Small positive changes were seen in their knowledge measure but the validity of the tool used remains doubtful.

There is insufficient data to answer the review question concerning how one might design a successful intervention. Unfortunately, no elements were found to be consistent between the studies with positive results. In the absence of this evidence it is tempting to extrapolate from similar adult studies (summarised in a review as showing positive results $^{19}$ ). However, two papers present findings that caution against this. 'In our own voice' had positive results in adults but 'disappointing' results in adolescents ${ }^{40}$ and a more recent meta-analysis of anti-stigma approaches reported that although 'contact' was better than education at reducing stigma in adults the reverse was true in adolescents. $^{21}$

Results from studies to date leave uncertainty as to whether interventions to reduce stigma in schools are not effective, whether interventions have been unsuccessful because they have not contained the right combination of elements or whether the studies have not been designed in such a way as to demonstrate efficacy.

Challenges in developing interventions include the need to assess different elements of programme content 
(contact, educational, etc.) and delivery style against each other. Information is also needed about whether targeting certain groups of children is more successful than universal provision. Indeed, not all students may need an intervention of this type. Only a third of pupils in a Scottish study reported moderate-high levels of stigma. ${ }^{41}$ It is also unclear whether the primary-school age child would be more open to anti-stigma messages, as very few studies have been carried out in this age group.

It is proposed here that the starting assumption when developing an intervention is that it should be long enough and intensive enough to provide some effect at follow-up. The studies reviewed here do not agree on how long a successful intervention should be or at what interval to assess follow-up.

There are daunting issues for study designers to contend with in this field. Stigma is a multifaceted concept, and even well-established measures have their limitations (for example social distance scales not being validated against discriminatory or supportive behaviour ${ }^{42}$ ). These measures are self-report questionnaires, which are at risk of social desirability bias (particularly, it could be argued, if done after an anti-stigma intervention). The absence of measures to examine change in behaviour after anti-stigma programmes has been recently commented on in a metaanalysis as regrettable. ${ }^{21}$ Maybe resources need to be first directed towards refining age-appropriate measures more closely linked to actual behavioural outcomes. Adverse effects of an intervention also need to be monitored. Recruiting pupils within a school environment is also challenging. Recruitment difficulties in some of the studies described led to a need to actively recruit volunteers to the intervention arm, leading to problems with selection bias. It is also resource heavy to expose the control group to a different type of intervention - hence most of the controls in these studies were simply exposed to 'normal lessons'.

The protocol of a proposed UK-based, feasibility trial $^{43}$ tackles many of these methodological issues. This well-powered study plans to have an active and randomised control (describing adequate sequence generation and allocation), comparing education with education and contact, carefully prepared material already piloted, 2 -week and 6-month follow-up, and will compare the intervention effect by baseline characteristics. ${ }^{43}$ If this trial does not suffer from significant implementation and reporting difficulties the results will be the most definitive to date. This review shows that, although it is inherently attractive to believe that school-based interventions reduce stigma to mental illness in young people, there is currently no strong evidence to support this conclusion.

\section{Limitations}

The limitations of this review include not searching the grey literature and the exclusion of studies written in foreign languages. There is a risk of bias in study selection and data extraction as one author performed these processes. Also, the authors of studies were not contacted for information that could not be gleaned from the published study papers.

\section{About the author}

Dr Catriona Mellor is a specialty trainee year 6 in child and adolescent psychiatry in Oxford Health NHS Foundation Trust.

\section{References}

1 World Health Organization. Atlas: Child and Adolescent Mental Health Resources: Global Concerns, Implications for the Future. WHO, 2005

2 Corrigan P, Kerr A, Knudsen L. The stigma of mental illness: explanatory models and methods for change. Appl Prev Psychol 2005; 11: 179-90.

3 Gulliver A, Griffiths KM, Christensen H. Perceived barriers and facilitators to mental health help-seeking in young people: a systematic review. BMC Psychiatry 2010; 10: 113.

4 Wahl OF. Children's views on mental illness: a review of the literature. Psychiatr Rehab Skills 2002; 6: 134-58.

5 Thornicroft G, Brohan E, Rose D, Sartorius N, Leese M, Group IS. Global pattern of experienced and anticipated discrimination against people with schizophrenia: a cross-sectional survey. Lancet 2009; 373: 408-15.

6 World Health Organization. The World Health Report 2001. Mental Health: New Understanding, New Hope. WHO, 2001.

7 National Institute of Mental Health. Translational Research: Bridging Basic and Applied Perspectives. NIMH, 2006.

8 Paykel ES, Hart D, Priest RG. Changes in public attitudes to depression during the Defeat Depression Campaign. Br J Psychiatry 1998; 173: 519-22.

9 Vaughan G, Hansen C. 'Like Minds, Like Mine': a New Zealand project to counter the stigma and discrimination associated with mental illness. Australas Psychiatry 2004; 12: 113-7.

10 Gaebel W, Zäske H, Baumann AE, Klosterkötter J, Maier W, Decker P, et al. Evaluation of the German WPA "program against stigma and discrimination because of schizophrenia-Open the Doors": results from representative telephone surveys before and after three years of antistigma interventions. Schizophr Res 2008; 98: 184-93.

11 Mehta N, Kassam A, Leese M, Butler G, Thornicroft G. Public attitudes towards people with mental illness in England and Scotland, 1994-2003. Br J Psychiatry 2009; 194: 278-84.

12 Department for Education and Skills. Excellence and Enjoyment: Social and Emotional Aspects of Learning. DfES, 2005.

13 Department for Education and Skills. National Healthy Schools Status A Guide for Schools. DfES, 2005.

14 National Institute for Health and Clinical Excellence. Promoting Young People's Social and Emotional Wellbeing in Secondary Education. NICE, 2009.

15 Department of Health and Department for Children, Schools and Families. Healthy Lives, Brighter Futures - The Strategy for Children and Young People's Health. Department of Health and Department for Children, Schools and Families, 2009.

16 Sakellari E, Leino-Kilpi $H$, Kalokerinou-Anagnostopoulou A. Educational interventions in secondary education aiming to affect pupils' attitudes towards mental illness: a review of the literature. J Psychiatr Ment Health Nurs 2011; 18: 166-76

17 Schachter HM, Girardi A, Ly M, Lacroix D, Lumb AB, van Berkom J, et al. Effects of school-based interventions on mental health stigmatization: a systematic review. Child Adolesc Psychiatry Ment Health 2008; 2: 18.

18 Ando S, Clement S, Barley EA, Thornicroft G. The simulation of hallucinations to reduce the stigma of schizophrenia: a systematic review. Schizophr Res 2011; 133: 8-16.

19 Dalky HF. Mental illness stigma reduction interventions: review of intervention trials. West J Nurs Res 2011; 34: 520-47.

20 Yamaguchi S, Mino Y, Uddin S. Strategies and future attempts to reduce stigmatization and increase awareness of mental health problems among young people: a narrative review of educational interventions. Psychiatry Clin Neurosci 2011; 65: 405-15.

21 Corrigan PW, Morris SB, Michaels PJ, Rafacz JD, Rüsch N. Challenging the public stigma of mental illness: a meta-analysis of outcome studies. Psychiatr Serv 2012; 63: 963-73. 
22 Cochrane Group. Suggested Risk of Bias Criteria for EPOC Reviews. Cochrane Group, no date (http://epoc.cochrane.org/sites/ epoc.cochrane.org/files/uploads/Suggested\%20risk\%20of\%20bias \%20criteria\%20for\%20EPOC\%20reviews.pdf).

23 Moher D, Liberati A, Tetziaff J, Altman D. Preferred reporting items for systematic reviews and meta-analyses: the PRISMA statement. BMJ 2009; 339: b2535.

24 Rahman A, Mubbashar MH, Gater R, Goldberg D. Randomised trial of impact of school mental-health programme in rural Rawalpindi, Pakistan. Lancet 1998; 352: 1022-5.

25 Esters IG, Cooker PG, Ittenbach RF. Effects of a unit of instruction in mental health on rural adolescents' conceptions of mental illness and attitudes about seeking help. Adolescence 1998; 33: 469-76.

26 O'Kearney R, Gibson M, Christensen H, Griffiths KM. Effects of a cognitive-behavioural internet program on depression, vulnerability to depression and stigma in adolescent males: a school-based controlled trial. Cogn Behav Ther 2006; 35: 43-54

27 O'Kearney R. A controlled trial of a school-based internet program for reducing depressive symptoms in adolescent girls. Depress Anxiety 2009; 26: 65-72.

28 Pitre N, Stewart S, Adams S, Bedard T, Landry S. The use of puppets with elementary school children in reducing stigmatizing attitudes towards mental illness. J Ment Health 2007; 16: 415-29.

29 Naylor PB, Cowie HA, Walters SJ, Talamelli L, Dawkins J. Impact of a mental health teaching programme on adolescents. $\mathrm{Br} J$ Psychiatry 2009; 194: 365-70.

30 Wahl OF, Susin J, Kaplan L, Lax A, Zatina D. Changing knowledge and attitudes with a middle school mental health education curriculum. Stigma Res Action 2011; 1: 44-53.

31 Ventieri D, Clarke D, Hay M. The effects of a school-based educational intervention on preadolescents' knowledge of and attitudes towards mental illness. Adv Sch Ment Health Promot 2011; 4: 5-17.

32 Economou M, Louki E, Peppou LE, Gramandani C, Yotis L, Stefanis CN Fighting psychiatric stigma in the classroom: the impact of an educational intervention on secondary school students' attitudes to schizophrenia. Int J Soc Psychiatry 2011; 58: 544-51.
33 Chan JY, Mak WW, Law LS. Combining education and video-based contact to reduce stigma of mental illness: "The Same or Not the Same" anti-stigma program for secondary schools in Hong Kong. Soc Sci Med 2009; 68: 1521-6.

34 Saporito J, Ryan C, Bethany A, Teachman B. Reducing stigma toward seeking mental health treatment among adolescents. Stigma Res Action 2011; 2: 9-21.

$35 \mathrm{Ng}$ P, Chan KF. Attitudes towards people with mental illness. Effects of a training program for secondary school students. Int J Adolesc Med Health 2002; 14: 215-24.

36 Schulze B, Richter-Werling M, Matschinger H, Angermeyer MC. Crazy? So what! Effects of a school project on students' attitudes towards people with schizophrenia. Acta Psychiatr Scand 2003; 107: 142-50.

37 Rickwood D, Cavanagh S, Curtis S, Sakrouge R. Educating young people about mental health and mental illness: evaluating a school-based programme. Int J Ment Health Promot 2004; 6: 23-32.

38 Conrad I, Dietrich S, Heider D, Blume A, Angermeyer M, Riedel-Heller S "Crazy? So what!": a school programme to promote mental health and reduce stigma - results of a pilot study. Health Educ 2009; 109: 314-28.

39 Robinson J, Gook S, Yuen H, Hughes A, Dodd S, Bapat S, et al. Depression education and identification in schools: an Australian-based study. School Ment Health 2010; 2: 13-22.

40 Pinto-Foltz MD, Logsdon MC, Myers JA. Feasibility, acceptability, and initial efficacy of a knowledge-contact program to reduce mental illness stigma and improve mental health literacy in adolescents. Soc Sci Med 2011: 72: 2011-9.

41 Chandra A, Minkovitz CS. Stigma starts early: gender differences in teen willingness to use mental health services. J Adolesc Health 2006; 38 754.e1-8.

42 Jorm $A F$, Oh $E$. Desire for social distance from people with mental disorders. Aust N Z J Psychiatry 2009; 43: 183-200.

43 Chisholm KE, Patterson P, Torgerson C, Turner E, Birchwood M. A randomised controlled feasibility trial for an educational school-based mental health intervention: study protocol. BMC Psychiatry 2012; 12: 23.

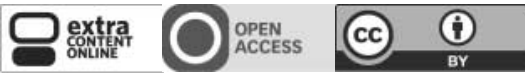

